\title{
Adrenal tumours in pregnancy: diagnostic challenge and management dilemma
}

\author{
Jessie Wai Leng $\underline{\text { Phoon }}^{1}$, MBChB, Mrcog, Devendra Kanalingam ${ }^{2}$, MBBs, Mrcog, Hong Liang $\underline{\text { Chua }}^{2}$, MBBS, MRcoG
}

\begin{abstract}
Adrenal gland tumours in pregnancy are very rare occurrences and have highly variable clinical presentations. The timely diagnosis of adrenal tumours in pregnancy is extremely important, as failure to do so may lead to fatality. As there is limited published literature on adrenal tumours in pregnancy and no consensus on its management, the management of such patients with regard to medical and surgical treatments, as well as timing of delivery, must therefore be individualised and carried out with multidisciplinary expertise. We present two cases of adrenal tumours in pregnancy, both with favourable outcomes and variable gestations. Our first and second cases discuss a large phaeochromocytoma and a cortisol-secreting adrenal cortical adenoma in pregnancy, respectively.
\end{abstract}

Keywords: adrenal cortical adenoma, adrenal tumours, Cushing's syndrome, phaeochromocytoma, pregnancy

\section{INTRODUCTION}

Adrenal tumours are very rare during pregnancy and have highly variable clinical presentations. Commonly an incidental finding, adrenal tumours in pregnancy must be diagnosed in a timely manner, as failure to do so may lead to fatality. The rarity of this condition poses great difficulty in the formulation of an optimum plan of management. Currently, there is limited published literature on adrenal tumours in pregnancy and no consensus on its management, especially with regard to the need for, and timing of, medical and surgical treatments, as well as the timing of delivery. We present two cases that highlight some of these difficulties.

\section{CASE REPORTS}

Case 1

A 31-year-old Chinese primigravida with a history of migraine presented with recurrent episodes of migraine and nausea with vomiting since 28 weeks of gestation. The patient required hospitalisation for each occurrence and was treated conservatively. At 35 weeks of gestation, she was admitted for an episode of near syncope with migraine, nausea and vomiting. At presentation, she was normotensive with a blood pressure (BP) of approximately 109/52 mmHg. Her blood pressure never exceeded $120 / 80 \mathrm{mmHg}$, but her blood investigations revealed mild elevation in transaminases, prompting ultrasonography (US) of the hepatobiliary system. US revealed a large, predominantly solid mass (widest diameter $8.8 \mathrm{~cm}$ ) in the right suprarenal region. Abdominal magnetic resonance (MR) imaging subsequently confirmed this to be a well-circumscribed, retroperitoneal mass in the right suprarenal region and no normal adrenal gland was seen separately (Figs. $1 \& 2$ ). Further workup demonstrated

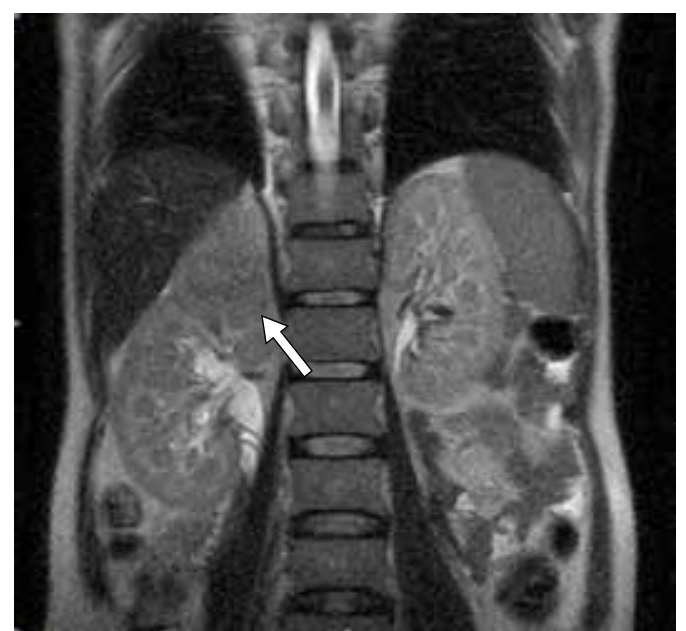

Fig. 1 Case 1: Coronal T2-W MR image shows a well-defined mass (arrow) in the right adrenal gland. The mass is slightly heterogeneous and isointense relative to the kidney.

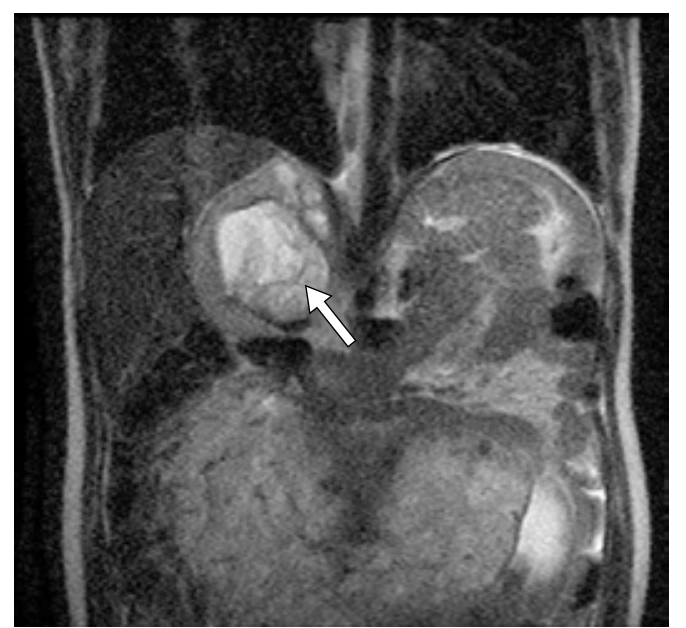

Fig. 2 Case 1: Coronal T2-W MR image shows a heterogeneous and predominantly hyperintense mass (arrow) in the right adrenal gland, indicating the presence of cystic/necrotic contents.

${ }^{1}$ Department of Obstetrics and Gynaecology, KK Women and Children's Hospital, ${ }^{2}$ Department of Obstetrics and Gynaecology, Singapore General Hospital, Singapore Correspondence: Dr Jessie Phoon, Medical Officer, Department of Obstetrics and Gynaecology, KK Women and Children's Hospital, 100 Bukit Timah Road, Singapore 229899. jess_phoon@yahoo.com 
Table I. Laboratory findings in Case 1.

\begin{tabular}{lc}
\hline Parameter & Result (NR) \\
\hline Urinary epinephrine* $(\mathrm{nmol} /$ day $)$ & $571(3-109)$ \\
Urinary norepinephrine* $(\mathrm{nmol} /$ day $)$ & $495(89-473)$ \\
Urinary metanephrine ${ }^{*}(\mathrm{nmol} /$ day $)$ & $18,261(400-1,500)$ \\
Urinary normetanephrine* $(\mathrm{nmol} /$ day $)$ & $13,663(600-1,900)$ \\
Urinary free cortisol* $(\mathrm{nmol} /$ day) & $213(59-413)$ \\
Serum plasma renin activity $(\mathrm{ug} / \mathrm{L} / \mathrm{hr})$ & $1.06(0.66-3.08)$ \\
Serum aldosterone $($ pmol/L) & $549.6(97.3-834)$ \\
\hline${ }^{*}$ Data presented is derived from 24-hour urine analysis. NR: normal range
\end{tabular}

marked elevation of 24-hour urinary catecholamines and metanephrines levels (Table I). The 24-hour urinary free cortisol and serum aldosterone/plasma renin activity were normal. The results of the investigations were suggestive of phaeochromocytoma.

As the patient had presented with suspected preterm labour, intramuscular steroids (two doses of dexamethasone $12 \mathrm{mg}$, given 12 hours apart) were administered to promote foetal lung maturity. The patient's BP remained normal throughout her pregnancy, with no paroxysmal hypertension. A preeclampsia workup, consisting of full blood count and coagulation profile, as well as creatinine, uric acid, urinary protein and liver function tests, was unremarkable. However, oral glucose tolerance test (OGTT) revealed impaired glucose tolerance, with venous glucose at $9.4 \mathrm{mmol} / \mathrm{L}$ two hours after oral glucose load. This was thought to be secondary to phaeochromocytoma. Foetal growth remained normal. A multidisciplinary approach, involving a senior obstetrician, a urologist, an endocrinologist and an anaesthetist, was applied. The patient was administered an alpha-blocker (phenoxybenzamine) in gradually increasing dosages over a period of approximately ten days (eventually reaching a dosage of $20 \mathrm{mg}$ twice daily [bd]). A beta-blocker (propranolol) at $10 \mathrm{mg}$ thrice daily (tds) was subsequently administered to prevent tachyarrhythmias. She also had fluid expansion with normal saline and was given sodium chloride tablets $2 \mathrm{~g}$ tds to avoid hypotension. She did not require any other antihypertensives.

We aimed for a planned delivery to avoid spontaneous labour or vaginal delivery. Planned delivery also allows for appropriate anaesthetic preparation such as intensive monitoring, placement of an intra-arterial line and central venous pressure access. It was thus planned that the patient would undergo an elective Caesarean section and laparoscopic adrenalectomy at term. However, she went into spontaneous labour at 36 weeks of gestation and an emergency lower segment Caesarean section (LSCS) under general anaesthesia had to be performed. LSCS was uneventful. Perioperatively, her haemodynamic parameters (BP, heart rate, saturation, central venous pressure) were monitored with arterial and central venous lines. Adrenalectomy was deferred in view of the emergency setting. Intraoperatively, systolic BP remained well below $160 \mathrm{mmHg}$ (range $110-152 \mathrm{mmHg}$ ), thus antihypertensives were not required.

The patient underwent laparoscopic adrenalectomy one month after LSCS. The surgery was converted into an open adrenalectomy in view of the large size of the tumour and the difficult surgical dissection. She remained stable intraoperatively and her postoperative recovery was uneventful. Histology confirmed the diagnosis of phaeochromocytoma. The result of the repeat OGTT at eight weeks post adrenalectomy was within normal limits $(4.9 \mathrm{mmol} / \mathrm{L})$.

\section{Case 2}

A 31-year-old Chinese woman (gravida 5, para 2) developed high BP from 15 weeks of gestation, and was treated with methyldopa $250 \mathrm{mg}$ bd and nifedipine LA $30 \mathrm{mg}$ every morning (om). She was well antenatally in her previous pregnancies and had no medical issues. At 23 weeks of gestation, she was admitted for BP monitoring. She also complained of severe facial, trunk and upper limb acne, and noticed significant weight gain over a period of one year, which was associated with swelling over the supraclavicular area and easy bruisability. Physical examination revealed rounded facies, increased supraclavicular and dorsolumbar fat deposition, and generalised, wide, pink striae. Severe acne and mildly atrophic skin was also observed. 24-hour urine analysis revealed proteinuria, with urinary total protein at $1.10 \mathrm{~g} /$ day. Blood investigations showed persistent hypokalaemia (3.0-3.2 mmol/L) and transaminitis (alanine transferase $60 \mathrm{U} / \mathrm{L}$, aspartate transaminase $67 \mathrm{U} / \mathrm{L}$, gamma-glutamyl transferase $129 \mathrm{U} / \mathrm{L}$ ). However, US of the hepatobiliary system was unremarkable. Cushing's syndrome was suspected, and the diagnosis was confirmed with further workup, which showed a loss of diurnal rhythm of cortisol with raised 24-hour urinary free cortisol (Table II).

MR imaging of the patient's abdomen confirmed the presence of a solitary, well-circumscribed left adrenal adenoma measuring $3.4 \mathrm{~cm} \times 2.8 \mathrm{~cm}$ (Figs. $3 \& 4$ ). Her care was jointly managed by a senior obstetrician, an obstetric medicine physician, an endocrinologist, a general surgeon and an anaesthetist. In view of the patient's preeclampsia, two doses of intramuscular dexamethasone $12 \mathrm{mg}$ were administered at 24 weeks of gestation in order to promote foetal lung maturity. She subsequently required nifedipine LA $60 \mathrm{mg}$ om, nifedipine LA $30 \mathrm{mg}$ every night and methyldopa $250 \mathrm{mg}$ tds for good control of BP. She also required subcutaneously administered insulatard 8 units bd and actrapid 6 units tds for the management of gestational diabetes mellitus during this period.

At 26 weeks of gestation, the possibility of carrying out a Caesarean section together with an adrenalectomy was 


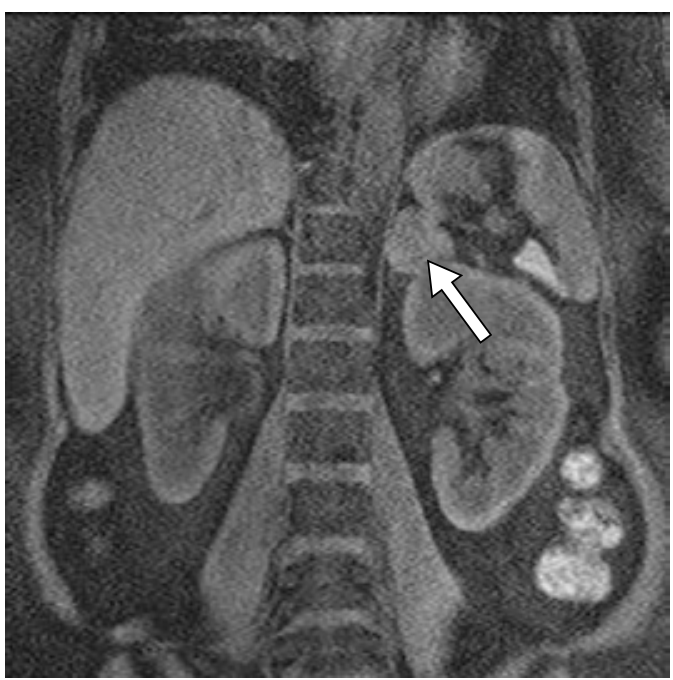

Fig. 3 Case 2: Coronal fat-saturated, post-contrast T1-W MR image shows a mildly enhancing, well-defined mass (arrow) in the left adrenal gland.

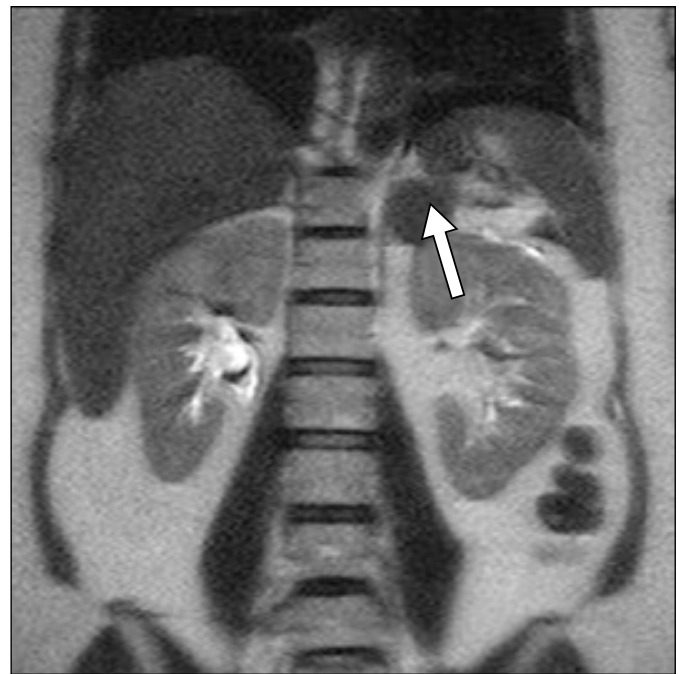

Fig. 4 Case 2: Coronal T2-W MR image shows a round, well-defined mass (arrow) in the left adrenal gland. The mass is hypointense relative to the kidney.

Table II. Laboratory findings in Case 2.

\begin{tabular}{lc}
\hline Parameter & Result (NR) \\
\hline Serum cortisol (nmol/L) & 776 \\
At 0000 hrs & 826 \\
At 0800 hrs & $2.2(10-60)$ \\
Serum ACTH (ng/L) & $3,163(59-413)$ \\
24-hour urinary free cortisol (nmol/day) & $16.8(\leq 7.8)$ \\
\hline
\end{tabular}

ACTH: adrenocorticotropic hormone; NR: normal range

considered in view of the patient's worsening preeclampsia, as evidenced by raised liver enzymes. Also, placental insufficiency was suspected based on umbilical arterial Doppler assessments. The decision on delivery was subsequently deferred when BP, liver enzymes and umbilical arterial Doppler studies remained stable, and in consideration of severe foetal prematurity. The patient was monitored closely as an inpatient. Eventually, she underwent an uneventful LSCS under general anaesthesia at 30 weeks of gestation. Postnatally, her BP was controlled with nifedipine LA $30 \mathrm{mg}$ om and she was started on metformin for glucose intolerance. Adrenalectomy was scheduled for a later date so as to avoid hypertensive crisis due to a prolonged surgery, and also as involution of her uterus after delivery would facilitate access to the adrenal tumour by laparoscopy. Her infant required inpatient neonatal care for 40 days for prematurity, but remained well on follow-up thereafter. An uneventful laparoscopic left adrenalectomy was performed six weeks after delivery. Histology revealed the mass to be an adrenal cortical adenoma. Two weeks post adrenalectomy, the patient's repeat OGTT was within normal limits $(6.6 \mathrm{mmol} / \mathrm{L})$. She no longer required antihypertensives or diabetes medications.

\section{DISCUSSION}

Adrenal tumours in pregnancy are very rare, and antenatal diagnosis of this condition is difficult and often missed. These two present cases illustrate several important aspects in the diagnosis and management of phaeochromocytoma and adrenal cortical adenoma. It is important to understand the histological distinction between these two tumours and their effects on pregnancy, as knowledge of the adverse effects and natural history of each tumour is the key to management.

In pregnancy, a BP above 140/90 $\mathrm{mmHg}$ is diagnosed as gestational hypertension. In clinically healthy pregnant women, BP steadily decreases till the middle of gestation and then increases up to the day of delivery, with final BP values that are similar to those found during early pregnancy in the same women. ${ }^{(1)}$ Most pregnant women with phaeochromocytomas present with symptomatic hypertension that fluctuates and is often severe, with associated headache, perspiration, palpitation and tachycardia. ${ }^{(2)}$ Oliva et al suggested screening for phaeochromocytoma only when there is persistence of symptoms and a failure to lower BP despite multidrug maximal therapy. ${ }^{(3)}$ Case 1 is thus an unusual presentation for a phaeochromocytoma, as maternal hypertension was absent. Other reported signs and symptoms that were absent in the patient include arrhythmias, postural hypotension, chest or abdominal pain, visual disturbance, convulsions, and sudden collapse.

The coexistence of glucose intolerance (as in Cases 1 and 2) and hyperthyroidism are known associations of phaeochromocytoma. (2) When phaeochromocytoma is suspected, the diagnosis can be confirmed by the presence of excessive catecholamine production, followed by imaging to detect the adrenal tumour. The most sensitive tests would, however, be measurements of plasma free metanephrines or urinary metanephrines. ${ }^{(4,5)}$ Laboratory diagnosis of phaeochromocytoma is largely unchanged from that in the nonpregnant state, as catecholamine metabolism is not altered by pregnancy. ${ }^{(6,7)}$ In Case 1 , the 
diagnosis was fortuitous, as US visualisation of the adrenal tumour was primarily intended for evaluation of the patient's liver. With the increasing utilisation of imaging studies, approximately $25 \%$ of phaechromocytomas are diagnosed incidentally. ${ }^{(8)}$ This underscores an important aspect of the modern management of these tumours. US is a valuable diagnostic tool in pregnancy, and when used to detect lesions of the adrenal glands, it has a reported sensitivity rate of $89 \%-97 \%{ }^{(9)}$ Computed tomography is commonly used to localise adrenal masses and detect extra-adrenal or metastatic disease, with $94 \%$ sensitivity for tumours measuring more than $1 \mathrm{~cm}$. However, in pregnancy, MR imaging is preferred so as to minimise radiation exposure. ${ }^{(10)}$ Gadolinium, a United States Food and Drug Administration Category C agent, is usually contraindicated in pregnant women, and thus non-contrast-enhanced MR imaging is preferred. Adrenal phaeochromocytoma demonstrates a wide range of appearances on T2-weighted (T2-W) MR imaging. In particular, a heterogeneously bright or hyperintense lesion on T2-W image (Fig. 2) is a useful diagnostic feature of phaeochromocytoma.

In Case 2, a diagnosis of Cushing's syndrome secondary to a cortisol-secreting adrenal adenoma was made. This condition rarely occurs in pregnant women, as hypercortisolism results in ovulatory disturbances and relative infertility. ${ }^{(2)}$ So far, only slightly more than 100 cases of Cushing's syndrome in pregnancy have been reported in the literature. It is thus unsurprising that this condition in pregnant women is difficult to detect clinically, as the classical signs of central weight gain, abdominal striae, increased blood pressure and glucose intolerance are also frequently associated with pregnancy. A high index of suspicion is necessary in order to make the diagnosis, as evident in Case 2, in which the obstetric medicine physician had felt that the clinical signs were more florid than could be attributed to physiological pregnancy changes. It is important to note that a benign adrenal tumour is more likely to be the cause of Cushing's syndrome in pregnant women as compared to non-pregnant women in whom pituitary-dependent disease predominates. Laboratory testing for Cushing's syndrome in pregnancy can be problematic, as there are no established reference ranges for cortisol in pregnancy. Random measurement of serum or urinary cortisol is not useful, as the levels found in a normal pregnancy may overlap with those found in Cushing's syndrome. The normal circadian rhythm of plasma cortisol, with high levels in the early morning and a nadir at night, is preserved in a healthy pregnant woman. As this rhythm is often lost in patients with Cushing's syndrome, it could be useful as a preliminary test. This was the case for the patient in Case 2.

The treatment of adrenal tumours during pregnancy is a clinical dilemma. Although the definitive treatment of adrenal tumour is surgical excision, there is scarce literature and little consensus on its management, with controversy surrounding the need for, and timing of, surgical and medical treatment, as well as the timing of delivery. Making the diagnosis is important, as treatment improves outcome. In some early studies where the diagnosis of phaeochromocytoma was often not known antenatally, very high maternal and foetal mortality rates $(48 \%$ and $54.4 \%$, respectively) were reported. ${ }^{(2)}$ Antenatal diagnosis and treatment, however, can reduce maternal mortality to $2 \%$, ${ }^{(2)}$ and medical management with an alpha-adrenergic blocker, such as phenoxybenzamine, has been found to be safe in pregnancy. Combined alpha- and beta-blockers, such as labetalol, have also been used in pregnancy with no adverse foetal effects. (2) The use of beta-blockers, which is important in preventing tachyarrythmia, should be kept to short-term usage in view of the possible risk of foetal growth restriction. ${ }^{(11)}$ Medical treatment of phaeochromocytomas is necessary in order to facilitate surgery, as performing surgery in an untreated state can be life-threatening. Many authors have proposed a cut-off time of 24 weeks of gestation when considering surgical excision during pregnancy. This is because a large uterine size makes abdominal exploration difficult. Surgical removal of the mass before 24 weeks of gestation usually has a good outcome, provided that medical therapy is adequate. ${ }^{(12)}$ After 24 weeks of gestation, it may be advisable to discuss with the patient about delaying the treatment until reasonable foetal maturity is achieved.(13) In Case 1, surgical treatment after delivery was the better option, as the patient had been diagnosed late in the third trimester.

In Cushing's syndrome, antenatal diagnosis is also important, as untreated disease is associated with increased maternal mortality, preterm delivery and perinatal mortality. As described in our two cases, we attempted delaying the deliveries till as near term as possible so as to allow foetal maturity. Higher dose steroids were also given to promote foetal lung maturity, as only a small amount of steroids in the maternal circulation reaches the foetal compartment in normal pregnancy, despite maternal hypercortisolism. This is because steroids are rapidly cleared from maternal plasma, and those that enter the throphoblast re-enter the maternal compartment instead of the foetal compartment.(2) The case for surgical excision of the adrenal tumour is stronger for Cushing's syndrome, in which the prognosis for pregnancy improves following adrenalectomy. Associated complications such as diabetes mellitus and preeclampsia may be avoided if adrenalectomy is performed early. ${ }^{(2)}$ However, in Case 2, these complications had already occurred, and the presence of established preeclampsia and placental insufficiency at the time of diagnosis indicated that it was necessary to deliver the foetus once initial medical treatment for diabetes mellitus and hypertension was effective.

The conditions of both patients in this report were wellcontrolled with medical treatment, and the outcomes for both mothers and babies were good. Planned Caesarean 
section is often preferred to spontaneous vaginal delivery in patients with phaeochromocytoma, based on the theoretical concern that bearing down efforts during labour could precipitate the release of catecholamines from the tumour. ${ }^{(2)}$ Planned delivery in an elective setting also confers the benefit of ideal preoperative preparation in preventing a fatal crisis. Although vaginal delivery is not contraindicated in the presence of Cushing's syndrome, the presence of preeclampsia, placental insufficiency and the need to deliver the foetus at 30 weeks neccessitated a Caesarean section. If adrenalectomy is to be performed after delivery of the foetus, this can be done as a one-stage procedure together with the Caesarean section or as an interval procedure after the puerperium, as was done in both our patients.

With the advent of minimally invasive procedures, laparoscopy has become the current surgical option of adrenalectomy, as opposed to a laparotomy. With decreased pain, shorter hospitalisation and recovery time, as well as improved patient satisfaction, laparoscopic adrenalectomy is the preferred approach for benign functioning or nonfunctioning tumours that measure less than $12 \mathrm{~cm}^{.(14)}$ Laparoscopic adrenalectomy, especially for phaeochromocytoma, is a safe and effective procedure. The complication rate is less than $8 \%$ and operative mortality rate is less than $1 \%$ in experienced centres. ${ }^{(15)}$ The laparoscopic approach was used in both our patients, although the patient in Case 1 eventually required a laparotomy due to the difficult operation.

In conclusion, as adrenal gland tumours are rare in pregnancy, the lack of familiarity with this condition may pose problems in diagnosis and management. It is thus crucial to understand the clinical presentations of these tumours and have a high index of suspicion. Our patients form a heterogeneous group as the actual tumour type, clinical presentation, gestation and associated medical complications, such as hypertension, diabetes mellitus and foetal compromise, differ. The management of these patients with regard to medical and surgical treatments, as well as timing of delivery, must therefore be individualised and carried out with multidisciplinary expertise. Our two cases demonstrate that when this is done, favourable outcomes are often possible.

\section{REFERENCES}

1. Hermida RC, Ayala DE, Mojón A, et al. Blood pressure patterns in normal pregnancy, gestational hypertension, and preeclampsia. Hypertension 2000; 36:149-58.

2. Abraham MR, Khardori R, Spandorfer SD, Talavera F. Adrenal disease and pregnancy. In: Endocrinology Articles (Pathophysiology, Symptoms, Causes, Diagnosis, Treatment, Medication, Surgery, Prognosis)-Medscape Reference [online]. Available at: http://emedicine.medscape.com/ endocrinology.

3. Oliva R, Angelos P, Kaplan E, Bakris G. Pheochromocytoma in pregnancy: a case series and review. Hypertension 2010; 55:600-6.

4. Lenders JW, Pacak K, Walther MM, et al. Biochemical diagnosis of pheochromocytoma: which test is best? JAMA 2002; 287:1427-34.

5. Sawka AM, Jaeschke R, Singh RJ, Young WF Jr. A comparison of biochemical tests for pheochromocytoma: measurement of fractionated plasma metanephrines compared with the combination of 24-hour urinary metanephrines and catecholamines. J Clin Endocrinol Metab 2003; 88:553-8.

6. George J, Tan JYL. Pheochromocytoma in pregnancy: a case report and review of literature. Obstet Med 2010; 3:83-5.

7. Jaffe RB, Harrison TS, Cerny JC. Localization of metastatic pheochromocytoma in pregnancy by caval catheterization. Including urinary catecholamine values in uncomplicated pregnancies. Am J Obset Gynecol 1969; 104:939-44.

8. Amar L, Servais A, Gimenez-Roqueplo AP, et al. Year of diagnosis, features at presentation, and risk of recurrence in patients with phaeochromocytoma or secreting paraganglioma. J Clin Endocrinol Metab 2005; 90:2110-6.

9. Shapiro B, Gross MD. Endocrine crises. Pheochromocytoma. Crit Care Clin 1991; 7:1-21.

10. Lyman DJ. Paroxysmal hypertension, pheochromocytoma, and pregnancy. J Am Board Fam Pract 2002; 15:153-8.

11. Butters L, Kennedy S, Rubin PC. Atenolol in essential hypertension during pregnancy. BMJ 1990; 301:587-9.

12. Ahlawat SK, Jain S, Kumari S, Varma S, Sharma BK. Pheochromocytoma associated with pregnancy: case report and review of the literature. Obstet Gynecol Surv 1999; 54:728-37.

13. Chandraharan E, Arulkumaran S. Pituitary and adrenal disorders complicating pregnancy. Curr Opin Obstet Gynecol 2003; 15:101-6.

14. Kuruba R, Gallagher SF. Current management of adrenal tumors. Curr Opin Oncol 2008; 20:34-46.

15. Lenders JW, Eisenhofer G, Mannelli M, Pacak K. Phaeochromocytoma. Lancet 2005; 366:665-75. 